\title{
Numerical Simulation of Water Flow through the Bottom End Piece of a Nuclear Fuel Assembly*
}

\author{
Moysés A. NAVARRO** and André A. C. dos SANTOS*** \\ ** Nuclear Technology Development Center - CDTN, Brazilian Nuclear Energy Commission - CNEN \\ Av. Antonio Carlos 6627, Pampulha, Belo Horizonte 31270-901, MG, Brazil \\ E-mail: navarro@cdtn.br \\ *** Department of Mechanical Engineering - DEMEC, Federal University of Minas Gerais - UFMG \\ Av. Antonio Carlos 6627, Pampulha, Belo Horizonte 31270-901, MG, Brazil \\ E-mail: acampagnole@yahoo.com.br
}

\begin{abstract}
An experimental and numerical study was conducted on the pressure loss of flows through the bottom end piece of a nuclear fuel assembly. To determine an optimized numerical methodology using the commercial CFD code, CFX 10.0, a series of preliminary simulations of water flows through perforated plates in a square ducts were performed. A perforated plate is a predominant geometry of the bottom end piece, responsible for the majority of the flow's pressure drop. The numerical pressure loss applying an optimized mesh and the $k-\varepsilon$ turbulence model showed good agreement when compared with a conventional methodology (Idelchik). Numerical results for the standard bottom end piece were obtained applying the previously determined mesh criteria and the $k-\varepsilon$ turbulence model with some geometric simplifications. The agreement between the numerical simulations and experimental results can be considered satisfactory but suggests further numerical investigations with the bottom piece under real conditions of the experiment, without the geometric simplifications and with a gap between the piece and the wall of the flow channel. Additionally, other turbulence models should be appraised for this complex geometry.
\end{abstract}

Key words: Nuclear Fuel Element, Bottom End Piece, Computational Fluid Dynamics (CFD), Perforated Plate, Pressure Loss, PWR

\section{Introduction}

The bottom end piece of a nuclear fuel assembly is constituted of a perforated plate and a flow nozzle. The perforated plate is the critical component of the end piece, being responsible for the majority of the flow's pressure loss. The extremities of the guide tubes of the fuel assembly are stuck by a nut on the perforated plate. Besides this structural function, the bottom perforated plate is a flow conditioner at the entrance of the fuel element. Recently, bottom end pieces manufactured with plates with small holes have been developed for an additional purpose of filtering debris.

Perforated plates have been studied as flow conditioners for measuring stations ${ }^{(1)(2)}$, as distillation trays to increase the heat and mass transfer between fluids ${ }^{(3)}$ and as flow controller emerging from diffuser ${ }^{(4)}$.

The agreement between the pressure loss coefficients of perforated plates obtained from some experiments ${ }^{(5)}$ and that defined by Idelchik ${ }^{(6)}$ are not always acceptable. 
More recently, due to the great progress in the computational capacity, numerical modeling of flows through complex geometries such as perforated plates have been accomplished. As a consequence of this progress the commercial CFD codes have been strongly improved.

Although a considerable amount of studies on numerical simulation of the flow through perforated plates have been made ${ }^{(7)(8)(9)}$ some issues related to turbulence models, near wall treatment and refinement of the mesh have still not been properly explained.

This work presents results of a numerical modeling of flow through different perforated plate in a square duct performed with the commercial code CFX 10.0 ${ }^{(10)}$. After mesh refinement studies, a suitable modeling was accomplished with a perforated plate similar to the bottom end piece of a nuclear fuel element. Finally, the whole end piece was simulated and this numerical result was compared with an experimental result.

\section{Nomenclature}

$d:$ Orifice diameter, $\mathrm{m}$

$D P$ : Static pressure loss across the plate, $\mathrm{Pa}$

$K$ : Pressure loss coefficient

$L:$ Mesh edge length, $\mathrm{m}$

$P$ : Pressure, $\mathrm{Pa}$

$q_{m}$ : Mass flow rate, $\mathrm{kg} / \mathrm{s}$

Re : Reynolds number

$T$ : temperature, ${ }^{\circ} \mathrm{C}$

$V:$ Mean duct velocity, $\mathrm{m} / \mathrm{s}$

$W:$ Velocity in the $\mathrm{z}$ direction

$y^{+}$: Dimensionless wall distance parameter

$\alpha$ : Angular division of the orifice, degree

$\rho:$ Density of the water, $\mathrm{kg} / \mathrm{m}^{3}$

\section{Subscripts}

\footnotetext{
$2 \mathrm{i}$ : between the taps 2 and $\mathrm{i}(\mathrm{i}=4$ to 7 )

$C F X$ : Numerical (with CFX)

$I$ : Idelchik
}

\section{Experimental Methodology}

Figure 1 shows the schematic diagram of the experimental apparatus used in the measurement of the pressure loss through the bottom end piece. The bottom end piece is shown in Fig. 2. Flow conditions established in the experiments are presented in Table 1.

Table 1 - Experimental conditions

\begin{tabular}{clll}
\hline Test & Flow rate $[\mathrm{kg} / \mathrm{s}]$ & Pressure $[\mathrm{bar}]$ & Temperature $\left[{ }^{\circ} \mathrm{C}\right]$ \\
\hline 1 & $16.73 \pm 0.50$ & $0.53 \pm 0.04$ & $40.1 \pm 1.1$ \\
\hline 2 & $49.70 \pm 0.50$ & $1.04 \pm 0.04$ & $40.9 \pm 1.1$ \\
\hline 3 & $82.51 \pm 0.82$ & $1.93 \pm 0.04$ & $41.4 \pm 1.1$ \\
\hline 4 & $115.52 \pm 1.15$ & $3.48 \pm 0.04$ & $40.2 \pm 1.1$ \\
\hline 5 & $115.08 \pm 1.15$ & $3.54 \pm 0.04$ & $55.9 \pm 1.1$ \\
\hline
\end{tabular}


The experiments were performed with a water flow in a $0.23 \times 0.23 \times 1.41 \mathrm{~m}$ square duct. The uncertainty in the width of the duct was not evaluated, but from the construction project it could reach up to $+0.75 \mathrm{~mm}$. The measurements of the pressure drop at the positions shown in Fig. 1 were performed by differential transmitters. An uncertainty of about $7 \%$ of the value was found for the pressure loss obtained at the lowest flow rate and $\sim 1 \%$ for the remaining.

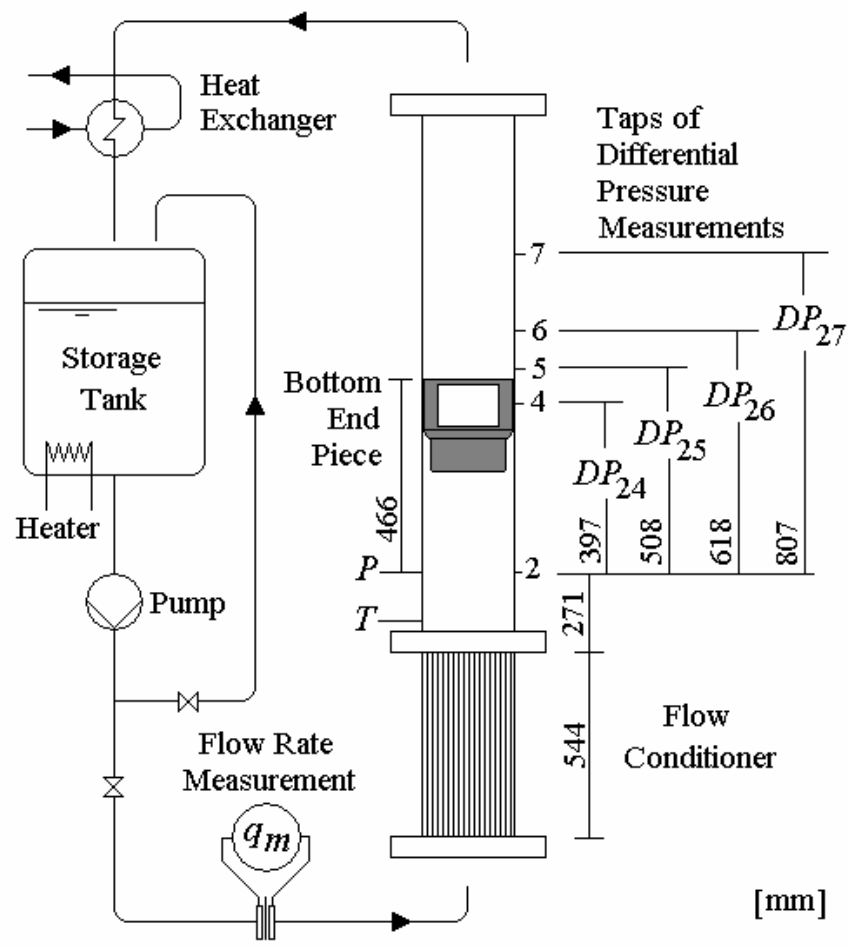

Fig. 1 Experimental apparatus.

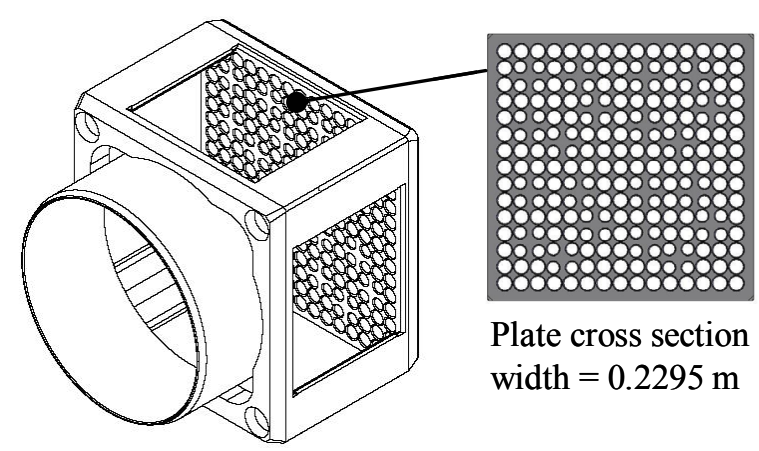

Fig. 2 Bottom end piece.

\section{The Numerical Modeling}

The numerical modeling was performed using the commercial code CFX 10.0 ${ }^{(10)}$. Water flows through perforated plates within a square duct were simulated under the experimental conditions established in test 2 (Table 1). The $k-\varepsilon$ turbulence model ${ }^{(11)}$ based on the Reynolds Averaged Navier-Stokes (RANS) equations was used in the simulations. Different mesh refinements were also evaluated. 
The pressure loss coefficient, $K$, for the perforated plate is defined by Eq. (1).

$$
K=\frac{D P}{(1 / 2) \rho V^{2}}
$$

The obtained $K$ for the numeric simulations were compared to values determined according to section 8 of the Handbook of Hydraulic Resistance by Idelchik ${ }^{(6)}$.

The DP assumed on $K$ determination for the perforated plate is the difference between the average static pressure at the same upstream (tap 2) and downstream (tap 7) positions used in the experiments with the bottom end piece minus the pressure loss due to friction on the duct wall.

\subsection{Model Definition}

To perform a mesh optimization without a great computational effort, a perforated plate with 81 holes $(9 \times 9)$ was used. As $\sim 70 \%$ of the pressure drop on the bottom end piece (BEP) occurs in its perforated plate a similar plate, with 225 orifices, was also evaluated. Table 2 shows the geometric details of the square array distribution of the orifices on the perforated plates used in the numerical simulations.

Table 2 - Details of the perforated plates

\begin{tabular}{cccc}
\hline \multicolumn{2}{c}{ Number of orifices } & $d[\mathrm{~mm}]$ & pitch [mm] \\
\hline \multirow{2}{*}{ Plates } & $81(9 \times 9)$ & 18.88 & 25.50 \\
& $225(15 \times 15)$ & 11.33 & 15.30 \\
\cline { 2 - 3 } BEP $^{1}$ & 80 & 10.00 & 14.30 \\
\cline { 2 - 3 } & 145 & 12.00 & \\
\hline
\end{tabular}

1 - Plate of the Bottom End Piece

The same plate thickness of $20 \mathrm{~mm}$ and perforation ratio of 0.43065 , of the plate of the BEP were used in all the plates. In the simulations the flow channel was a $0.2295 \times 0.2295$ $\mathrm{m}$ square duct with $0.7 \mathrm{~m}$ upstream and $0.71 \mathrm{~m}$ downstream lengths and the flow direction was in the $\mathrm{z}$ axis with the zero position was in the lower face of the plates.

To reduce the mesh size a symmetry slice of $1 / 8$ was applied to the geometries as shown in Fig. 3.

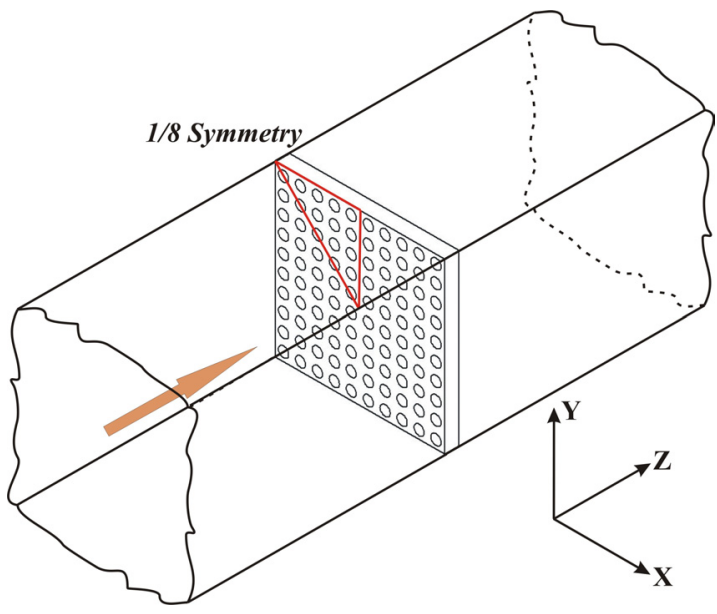

Fig. 3 Flow duct with a perforated plate. 
Aiming to evaluate the influence of the chamfers on both ends of the holes a simulation with the 225 orifice plate and a chamfer of $1 \mathrm{~mm} / 45^{\circ}$, similar to the plate of the bottom end piece was performed.

Figure 4 shows the geometric simplifications assumed for the bottom end piece. The simplifications were basically the removal of the bevel in the external corners. The reduction of the flow duct width from $0.23 \mathrm{~m}$ to the same width of the bottom end piece $0.2295 \mathrm{~m}$ was another simplification adopted. Both simplifications had the objective to reduce the mesh dimension. The four positioning orifices were also eliminated in the numerical simulations. As in the plate's simulations, the flow direction is in the $z$ axis, perpendicular to the plate of the BEP with the zero position in its lower face.

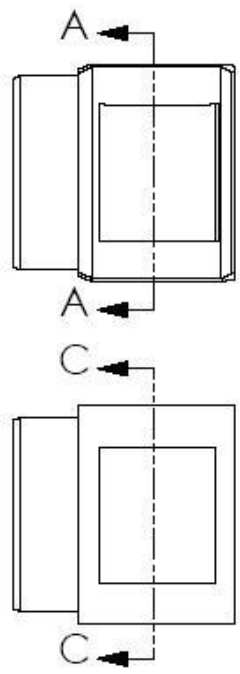

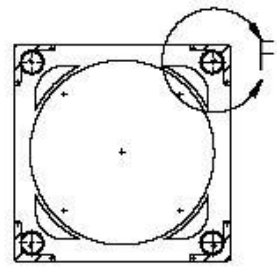

SECTION A-A

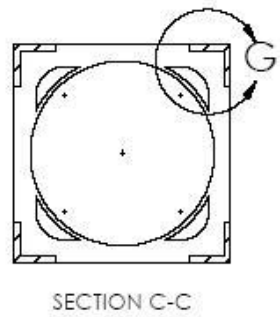

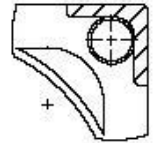

DETAIL F

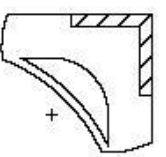

DETAIL G

Fig. 4 Simplifications in the bottom end piece geometry.

\subsection{Boundary Conditions}

The same flow conditions obtained in the BEP test number 2 were adopted in the simulations with the perforated plates. The simulations with the BEP were performed with all the five conditions shown in Table 1. In these simulations, the flow rates were reduced proportionally to the flow area reduction from the experimental flow duct $(0.23 \times 0.23 \mathrm{~m})$ to the numerical simulation model $(0.2295 \times 0.2295 \mathrm{~m})$.

The surfaces of the duct and plate were considered smooth and the inlet turbulence intensity was assumed to be 5\% (although simulations made showed no significant influence on the pressure drop when values of 1 and $10 \%$ of turbulent intensity were applied). A uniform velocity profile was assumed at the inlet of the flow channel. No thermal model was used since the experiments were considered adiabatic and isothermal.

\subsection{Turbulence Modeling}

The eddy viscosity two equation $k-\varepsilon$ turbulence model was used in the simulations. The $k-\varepsilon$ model, developed by Launder and Spalding ${ }^{(11)}$, assumes that the turbulence viscosity is related to the turbulence kinetic energy and dissipation. In CFX the $k-\varepsilon$ model uses a scalable wall-function approach to improve the near wall treatment which is made with the log-wall function. The basic idea behind the scalable wall function is to limit a lower value for the dimensionless distance from the wall used in the log-law in such a way that all the mesh points are outside the viscous sub layer. In this way all fine mesh inconsistencies near the wall can be avoided. 


\subsection{Numerical Scheme}

The numerical schemes applied for the simulations were second-order central differenced scheme for the diffusion terms and a high resolution (formally second-order) upwind scheme for the convective terms. The high resolution scheme developed by Barth and Jesperson ${ }^{(12)}$ reduces numerical diffusion and dispersion by evaluating the scheme locally applying the highest order possible, between first and second-order, that does not violate the boundedness principles.

\subsection{Mesh Definition}

In the CFX-Mesh the algorithms Delaunay Mesher and Advancing Front Mesher were used to generate the surface and the volumetric mesh, respectively. The global mesh scaling adopted in all simulations was of $16.5 \mathrm{~mm}$. Mesh controls were used to refine the superficial and volumetric mesh in specific regions. The perforated plate region was refined to better capture the rapid flow contraction and expansion. A refinement plane (Triangle Control), with a radius of influence of $10 \mathrm{~mm}$ was defined in the middle height of the plates. In the orifices region the angular division of an orifice $(\alpha)$, as shown in Fig. 5, was used as a parameter to determine the mesh edge length $(L)$ by Eq. (2).

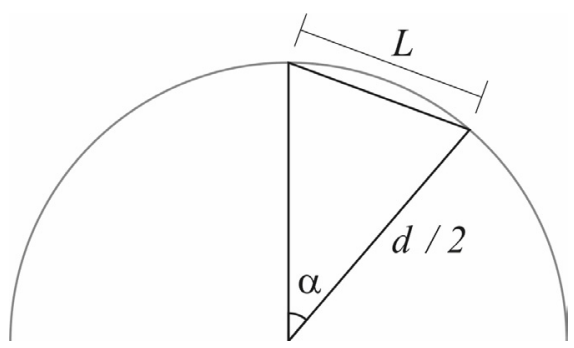

Fig. 5 Mesh edge length adopted in the orifice region

$$
L=d \operatorname{sen}(\alpha / 2)=0.5 d \sqrt{2-2 \cos \alpha}
$$

Some simulations were performed on the perforated plate with 81 orifices applying different angular divisions according to Table 3. A smooth element expansion factor of 1.1 was applied to enhance accuracy.

Table 3 - Pressure loss coefficients for the different mesh refinements and turbulence models.

\begin{tabular}{|c|c|c|}
\hline Refinement $-\alpha / L$ [degree $/ \mathrm{mm}]$ & $K_{C F X}$ & $\left(K_{C F X}-K_{I}\right) / K_{I} \quad[\%]$ \\
\hline $12 / 1.974$ & 3.73 & -4.6 \\
\hline $9 / 1.481$ & 3.84 & -1.8 \\
\hline $6 / 0.988$ & 3.85 & -1.5 \\
\hline
\end{tabular}

The downstream region of the perforated plate was also refined to capture the pressure recuperation that occurs in this region. Another refinement plane (Triangle Control) was used and its position for each of the plates was defined after extensive simulations. It was also found that the abrupt recovery of the pressure occurs at about six orifice diameters downstream of the plate. An element expansion factor of 1.2 was assumed on this refinement plane. The $L / d$ ratio was kept about 0.15 for all simulations on this plane.

Near the walls a structured mesh (inflation) was used to capture the effects of the boundary layer. An effort was made to maintain the value of the first dimensionless wall 
parameter $\left(y^{+}\right)$less than 2 because the same mesh would be used to assess other turbulence models with wall treatments which require lower $y^{+}$values. Twenty layers were used with an element expansion factor of 1.2.

On the bottom end piece a third refinement plane was used to refine the bottom support region. On this plane an edge length of $4 \mathrm{~mm}$ with an element expansion factor of 1.2 was applied.

Details of the inflation near the wall and of the localized refinements on an axial central plane of the 81 orifice plate are shown in Fig. 6.

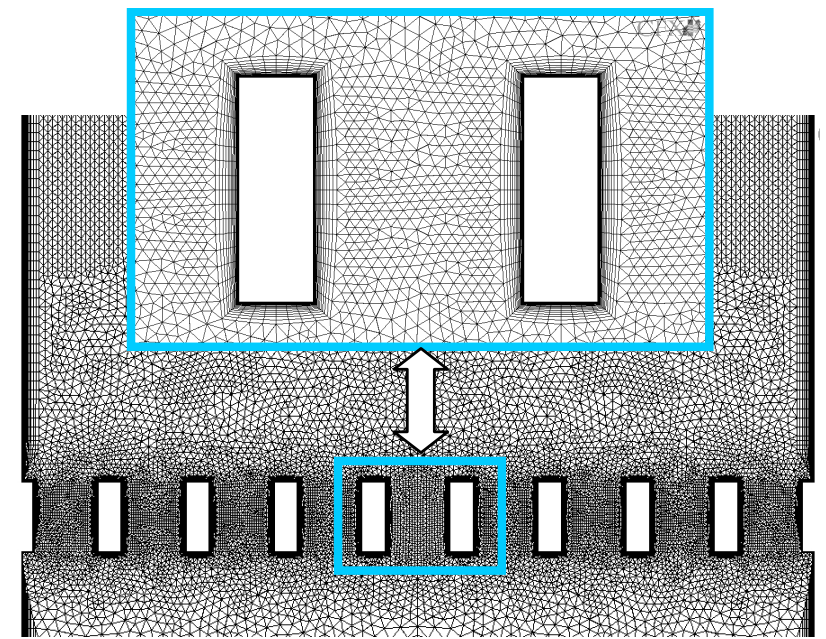

Fig. 6 Details of the mesh in the region of the plate with 81 orifices.

Table 3 shows the results obtained with different mesh refinements and turbulence models used in the numerical simulation with the 81 orifices plate. The pressure drop

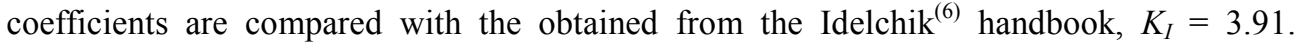
Table 3 shows clearly that there is little improvement of the results for edge lengths smaller than $1.5 \mathrm{~mm}$. Even so, the mesh refinement with an edge length of $0.988 \mathrm{~mm}$ was applied.

Figure 7 shows the static pressure behavior along a central line of the duct for different mesh refinements applied also on the 81 orifice plate. It can be seen that the pressure loss inside the holes increases as the mesh refinement increases and shows almost no difference for mesh's with edge lengths below $1.5 \mathrm{~mm}$.

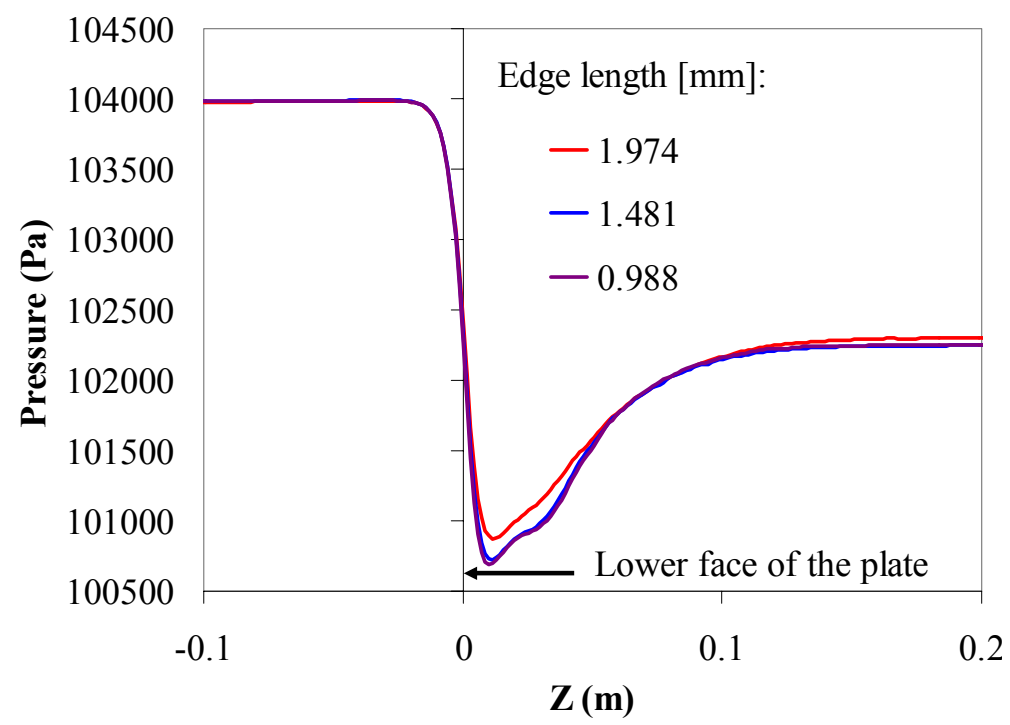

Fig. 7 Influence of the mesh refinement on the pressure drop. 
Details of velocity profile at entrance of a hole are shown in Fig. 8 for the refinements studied. It can be observed that the mesh with smaller edges captures better the flow detachment at the entrance of the hole and the steep velocity gradients downstream of the orifice.
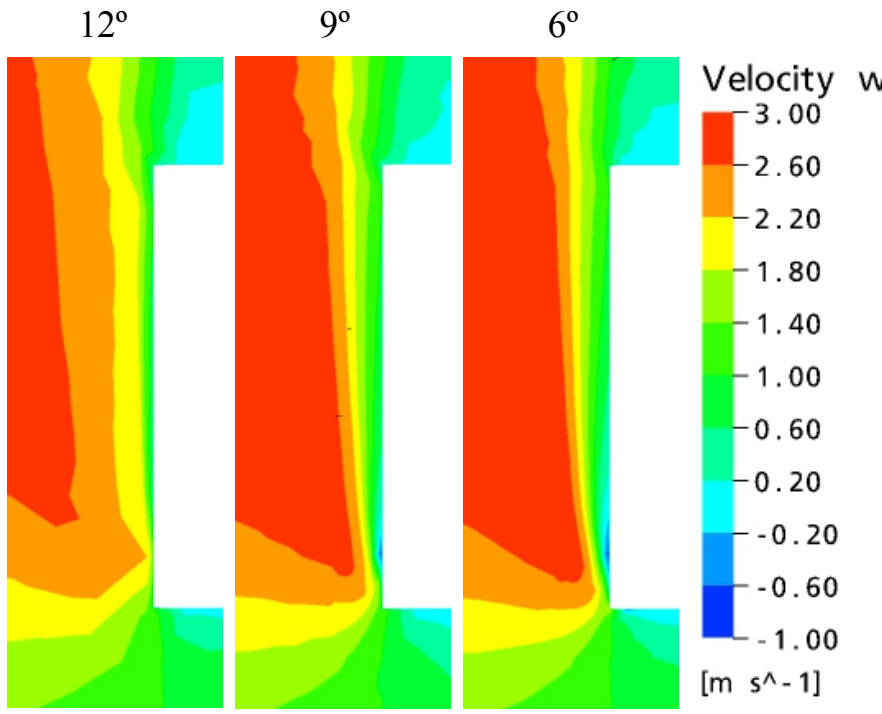

Fig. 8 Details of the velocity profile at the entrance of a hole for different edge lengths $(\alpha)$ for the plate with 81 holes.

The results of the simulations performed with the 81-hole plate led to the definition of the mesh parameters and turbulence model adopted for the simulations with the perforated plate with 225 holes. The mesh criterion remained the same and in the plate region the mesh edge length obtained with $\alpha=6^{\circ}$ in Eq. (2) was assumed. This was adopted to minimize the computational effort maintaining an acceptable mesh quality. This can be seen in Table 4 where the numbers of volumetric elements of all obtained meshes are shown. It can be observed that a more refined mesh would become too large and impossible to simulate with the available hardware. All simulations were performed though parallel runs on two Pentium 4 HT PCs with 4 GB of RAM memory. The simulations took $1.5\left(81\right.$ holes $\left.-12^{\circ}\right)$ to 7.5 (225 holes with chamfer) hours for the perforated plates and 15 hours for the BEP.

Table 4 - Number of volumetric elements.

\begin{tabular}{ccc}
\hline Plate/BEP & $\alpha$-[degree] & Number of elements \\
\hline \multirow{2}{*}{81} & 12 & 1154450 \\
& 9 & 1561866 \\
225 & 6 & 2306802 \\
$225^{1}$ & 6 & 6047291 \\
BEP & 6 & 6285987 \\
\hline
\end{tabular}

1 - Orifices with chamfer 


\section{Results}

Table 5 compares the pressure drop coefficients obtained in the numeric simulations with the Idelchik ${ }^{(6)}$ coefficients, for the perforated plates. In spite of the same perforation ratio for all of the plates, a reduction of the pressure loss coefficient with the decrease of the orifice diameter was verified. Figure 9 shows the profiles of the static pressure along the central line of the flow duct with different perforated plates. A reduction of the recovery pressure distance downstream the perforated plate with the decreasing of the orifice diameter was observed.

Table 5 - Pressure loss coefficients for perforated plates.

\begin{tabular}{cccc}
\hline Plate & $K_{C F X}$ & $K_{I}$ & Difference \% \\
\hline 81 & 3.85 & 3.91 & -1.53 \\
225 & 3.71 & 3.62 & 2.49 \\
$225^{1}$ & 2.03 & - & $-45.28^{2}$ \\
\hline
\end{tabular}

1 - Orifices with chamfer

2 - Relative to the numerical result without chamfer

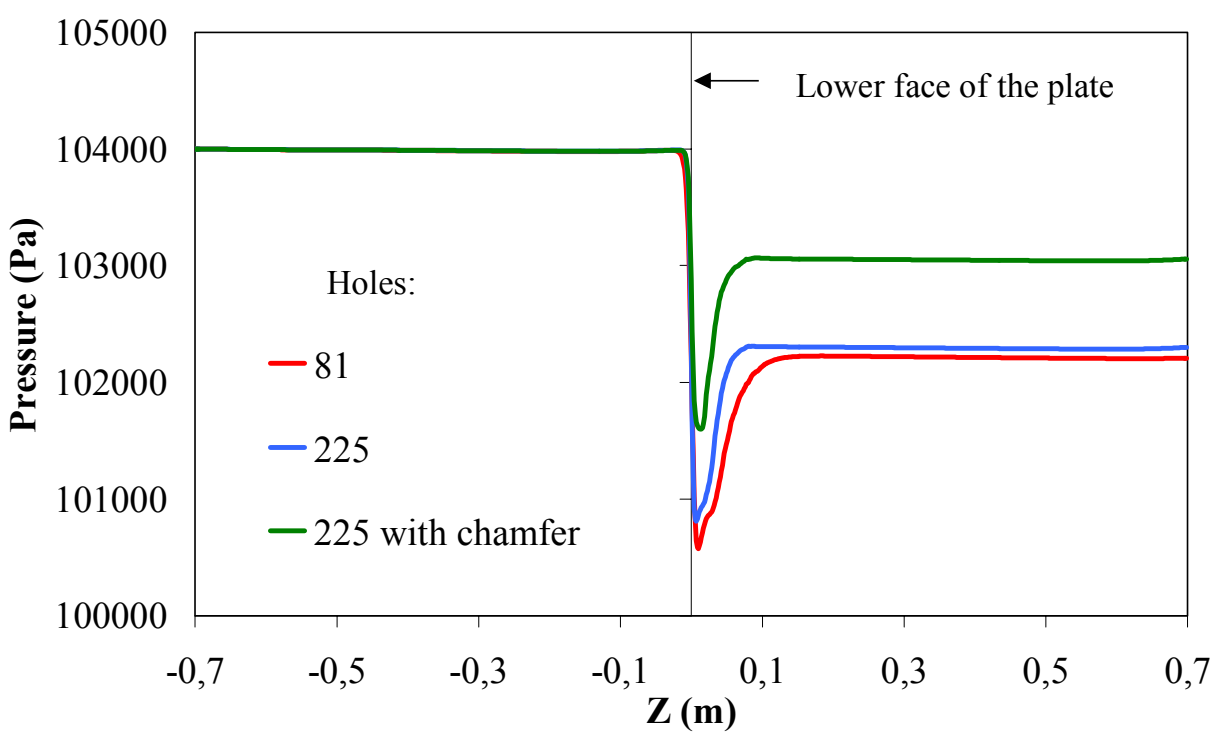

Fig. 9 Static pressure along the duct with different perforated plates.

A simulation was performed on a plate with 225 orifices with beveled edges on both ends, like the plate of the bottom end piece. As expected, the chamfer at the entrance of the orifice produces a smooth area reduction that leads to a lower pressure drop ( $45 \%)$ through the beveled plate, as shown in Fig. 9 and Table 5.

Figure 10 compares the velocity profiles in holes with and without chamfer. The separation observed at the entrance of hole without chamfer does not occur in the beveled hole. Additionally the velocity becomes larger near the wall of the hole. These observations explain the great reduction of the pressure loss observed when the chamfer was applied to the extremities of the holes. 

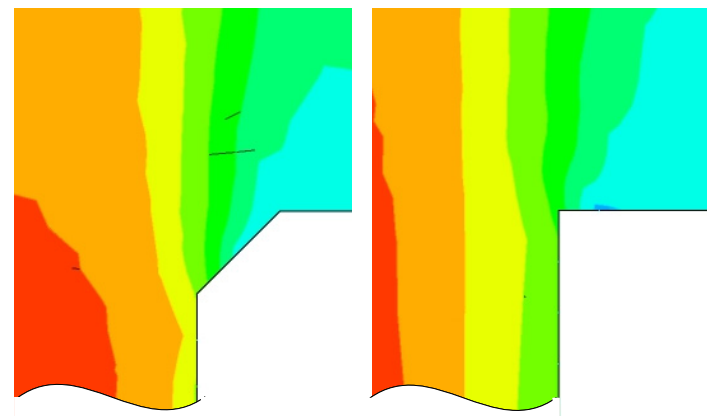

\section{Velocity w}
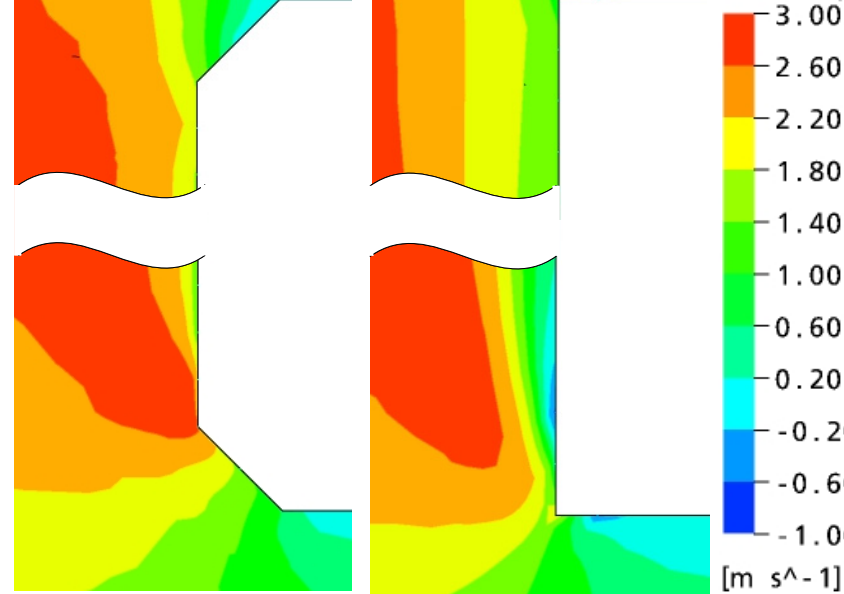

$-1.40$

$-1.00$

$-0.60$

$-0.20$

$-0.20$

$-0.60$

$-1.00$

[m $\left.s^{\wedge}-1\right]$

Fig. 10 Differences between the velocity profiles at an orifice with and without chamfer.

Figure 11 illustrates the velocity distribution found on the central plane of the bottom end piece for the test number 2 (Table 1).

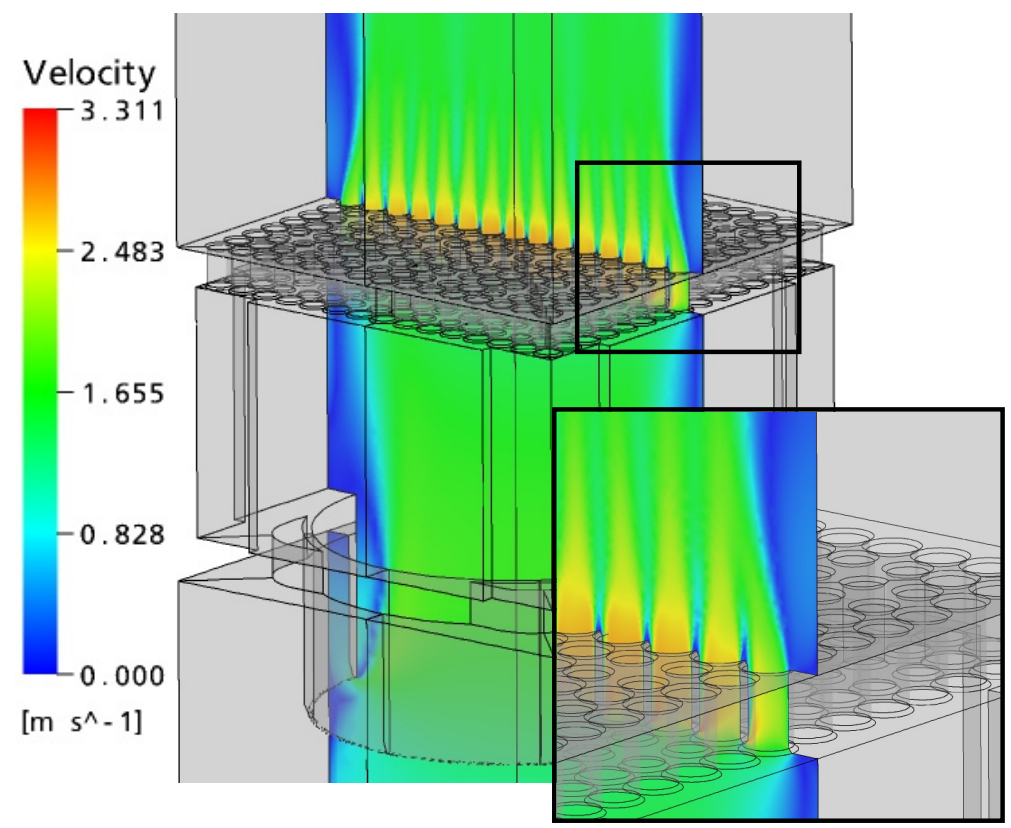

Fig. 11 Velocity distribution in a central plane of the bottom end piece.

Numerical and experimental pressure drop coefficients, obtained from the pressure difference between taps 2 and 7, are presented in Fig. 12 with the experimental uncertainties. The overall results show that the numerical pressure drop coefficients were slightly overestimated for the simulated flow rates. 


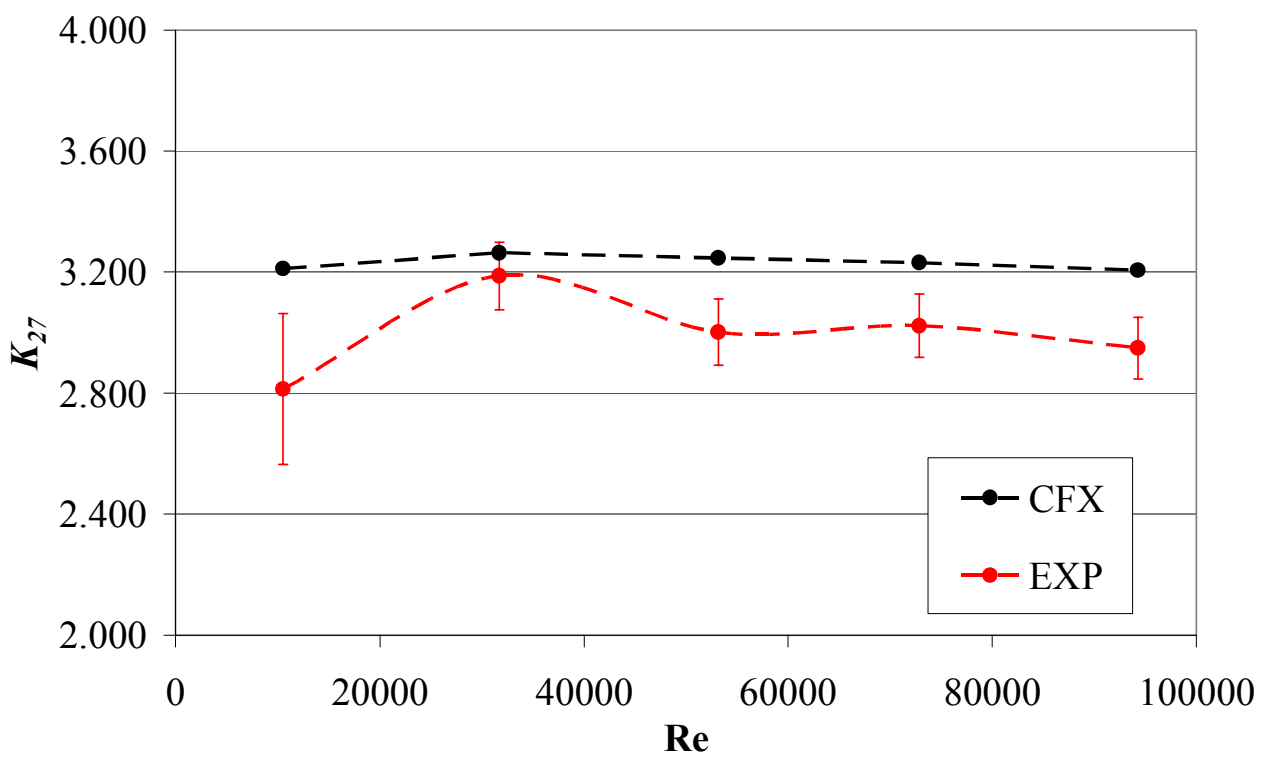

Fig. 12 Pressure loss coefficient $K_{27}$ for the BEP as a function of Re

The experimental and numerical results of the pressure loss along the duct obtained with the bottom end piece, normalized to the inlet average pressure are shown in Fig. 13. The numerical behavior of the pressure showed satisfactory agreement to the experiment, especially at the pressure recuperation region, tap 5 position.

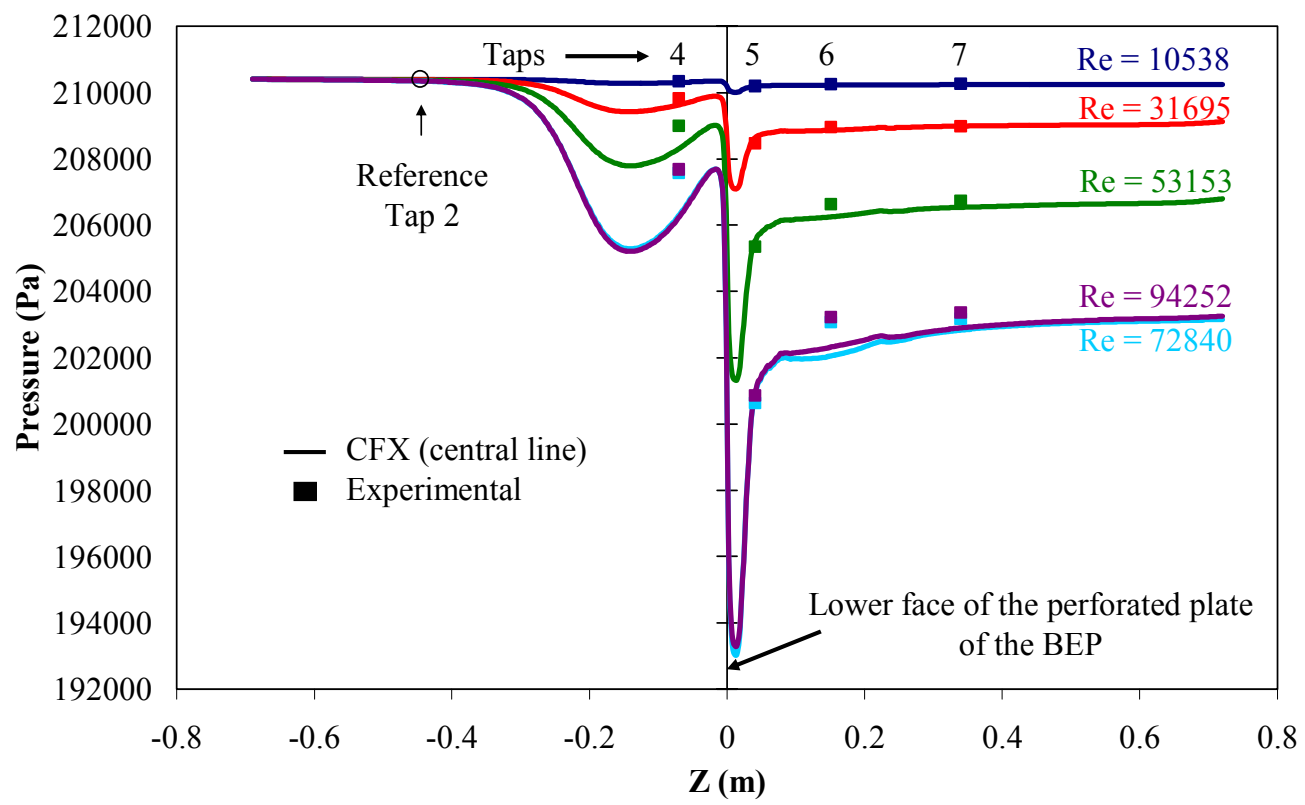

Fig. 13 Static pressure along the duct with the bottom end piece: experiments $\mathrm{x}$ numerical simulation.

\section{Conclusions}

In this analysis, experimental data concerning a pressure drop through a bottom end piece of a nuclear fuel element have been experimentally determined and compared to results from numerical simulations conduced with a commercial CFD code, CFX 10.0.

Previously, a numerical study was performed on a perforated plate with fewer holes to determine optimum mesh. The $k-\varepsilon$ turbulence model was used in the simulations. Mesh 
controls were used to refine the superficial and volumetric mesh in specific regions of the perforated plates to capture better the rapid flow contraction and expansion. The pressure drop coefficients obtained in these simulations were compared with those from Idelchik methodology and showed a good agreement. It was also verified that the chamfers on the ends of the holes generated a lower pressure drop through the plates.

The same mesh criterion and the $k-\varepsilon$ turbulence model were adopted in the bottom end piece simulations. Same simplifications were assumed in the geometric form of the piece which was responsible for some discrepancies obtained in the results. Limitations on the computational capacity disabled an appropriated simulation of the gap between the bottom end piece and the flow duct wall which can reach up to $0.625 \mathrm{~mm}$. Further investigations are still necessary to evaluate influence of the gap and of the real inlet velocity profile on the flow behavior.

Other turbulence models need to be appraised in simulations of complex geometries such as the bottom end piece.

\section{Acknowledgment}

The authors express their appreciation to the Centro Tecnológico da Marinha (CTMSP) and to the Indústrias Nucleares do Brasil (INB) for the experimental support to this research.

\section{References}

(1) Schlüter, Th. and Merzkirch, W., PIV measurements of the time-averaged flow velocity downstream of flow conditioners in a pipeline, Flow Measurement and Instrumentation, Vol. 7 (1996), pp. 173-179.

(2) Spearman, E. P., Sattary J. A. and Reader-Harris, M. J., Comparison of velocity and turbulence profiles downstream of perforated plate flow conditioners, Flow Measurement and Instrumentation, Vol. 7 (1996), pp. 181-199.

(3) Lockett, M. J., Distillation tray fundamentals, Cambridge Press, UK (1986).

(4) Sahin, B. and Ward-Smith, A. J., The use of perforated plates to control the flow emerging from a wide-angle diffuser, with application to electrostatic precipitator design, International Journal of Heat and Fluid Flow, Vol. 8 (1987), pp. 124-131.

(5) Gan, G. and Riffat, S. B., Pressure loss characteristics of orifice and perforated plates, Experimental Thermal and Fluid Science, Vol. 14 (1997), pp. 160-165.

(6) Idelchik, I. E., Handbook of hydraulic resistance, AEC-TR-6630 (1960), pp. 305-349.

(7) Erdal, A. and Andersson, H. J., Numerical aspects of flow computation through orifices, Flow Measurement and Instrumentation, Vol. 8 (1997), pp. 27-37.

(8) Erdal, A., A numerical investigation of different parameters that affect the performance of a flow conditioner, Flow Measurement and Instrumentation, Vol. 8 (1997), pp. 93-102.

(9) Frattolillo, A. and Massarotti, N., Flow conditioners efficiency a comparison based on numerical approach, Flow Measurement and. Instrumentation., Vol. 13 (2002), pp. 1-11.

(10) CFX-10.0, User manual, ANSYS-CFX (2005).

(11) Launder, B. E. and Spalding, D. B., The numerical computation of turbulent flow, Computer Methods in Applied Mechanics and Energy, Vol. 3 (1974), pp. 269-289.

(12) Barth, T. J. and Jesperson, D. C., The design and application of Upwind schemes on unstructured meshes, American Institute of Aeronautics and Astronautics Journal, Paper 89-0366 (1989). 